\title{
Charge qubit dynamics in a double quantum dot coupled to phonons
}

\author{
Zhuo-Jie Wu因 Ka-Di Zhu, Xiao-Zhong Yuan, Yi-Wen Jiang, and Hang Zheng \\ Department of Physics, Shanghai Jiao Tong University, Shanghai 200240, China
}

(Dated: June 24, 2018)

\begin{abstract}
The dynamics of charge qubit in a double quantum dot coupled to phonons is investigated theoretically in terms of a perturbation treatment based on a unitary transformation. The dynamical tunneling current is obtained explicitly. The result is compared with the standard perturbation theory at Born-Markov approximation. The decoherence induced by acoustic phonons is analyzed at length. It is shown that the contribution from deformation potential coupling is comparable to that from piezoelectric coupling in small dot size and large tunneling rate case. A possible decoupling mechanism is predicted.

PACS numbers: 73.23.Hk 73.63.Kv 03.65.Yz 03.67.Lx
\end{abstract}

\section{INTRODUCTION}

Computers based on quantum mechanics is proven, by computational theory, more efficient at some specific calculations than those based on classical physics $\stackrel{1.2 .3}{=}$ The first step to build a quantum computer is the realization of its building block, quantum bit (qubit). Within the last decade, various schemes have been proposed and many of them have even been realized 4.5,6.7.8 Among them, the electrically controlled charge qubit in a semiconductor double quantum dot has the potential advantages of being arbitrarily scalable to large system and compatible with the current microelectronics technology. Besides, the double-dot system is also extremely useful in basic physics as it enables us to investigate the decoherence and dissipation of a small quantum system interacting with its environment.

Various designs of double-dot qubits have been studied in experiment $\frac{9.10,11.12}{}$ Recently, Hayashi et al. successfully realized coherent manipulation of electronic states in a double-dot system implemented in a GaAs/AlGaAs heterostructure containing a two dimensional electron gas. The damped Rabi oscillation was observed in the time domain in their experiment and an experienced formula was presented to fit the experimental data 13 Fujisawa et al. explained the transport in this system through density matrix simulations ${ }^{14}$ But the phonon effect was not included. However, the phonon induced decoherence is significant according to the analysis of Ref. [13]. Considering the interaction with the piezoelectric acoustic phonons, Brandes et al. investigated in detail the single electron tunnelling in a double quantum dot 15,16.17.18,19 Fedichkin and Fedorov also studied the error rate of the charge qubit coupled to an acoustic phonon reservoir $\stackrel{20.21}{ }$ But no dynamical tunneling current is presented by all these work. In a latest review paper, ${ }^{22}$ Brandes derived the dynamical tunneling current in the weak electron-phonon dissipation limit through Born-Markov approximation (PER). But the result is rather complicated.

In this work, we study the damped Rabi oscillation observed in Ref. [13]. The quantum dynamics of a single electron tunneling in the double-dot system is investigate without applying the Born-Markov approximation to the electron-phonon interaction. A simple explicit expression of dynamical tunneling current is presented through perturbation treatment based on a unitary transformation. The phonon (both the deformation potential and piezoelectric contribution are included) induced decoherence is investigated at length and possible decoupling mechanism is presented.

This paper is organized as follows. In Sec. II we introduce the model Hamiltonian for the charge qubit and solve it in what follows. A comparison with PER approach is given in Sec. III. In Sec. IV, we derive the spectral density of a double quantum dot and then analyze the phonon induced decoherence. The conclusions is given in the Sec. $\mathrm{V}$ at last.

\section{MODEL AND THEORY}

In this section, we shall introduce the model Hamiltonain for the double quantum dot and then develop general theory leading to explicit expression for the dynamical current. We will focus on the phonon effect on qubit dynamics.

\section{A. Model Hamiltonian}

The double quantum dot consists of left and right dots connected through an interdot tunneling barrier. Due to Coulomb blockade, only one excess electron is allowed to occupy the left or right dot, which defines two basis vectors of $|L\rangle$ and $|R\rangle$ (with the energy level $\varepsilon_{L}$ and $\varepsilon_{R}$ respectively) in the Hilbert space. The energy difference between these two states $\varepsilon=\varepsilon_{L}-\varepsilon_{R}$ can be controlled by the source-drain voltage $V_{s d} \stackrel{13}{ }$ Considering the coupling to its environment, the double dot can be described by the Hamiltonian:

$$
H=H_{e}+H_{p}+H_{e p}+H_{r}+H_{e r} .
$$

Here the qubit Hamiltonian $\operatorname{reads}(\hbar=1)$

$$
H_{e}=-\frac{1}{2} \varepsilon(t) \sigma_{z}+T_{c} \sigma_{x},
$$


where $T_{c}$ is the interdot tunneling, $\sigma_{x}$ and $\sigma_{z}$ are Pauli matrix with $\sigma_{z}=|L\rangle\langle L|-| R\rangle\langle R|$ and $\sigma_{x}=|L\rangle\langle R|+$ $|R\rangle\langle L|$. If the qubit isolates from any other degrees of freedom, the excess electron would oscillate coherently between two dots with the Rabi frequency $\Delta=$ $\sqrt{\varepsilon^{2}+4 T_{c}^{2}}$. However, the qubit has to couple to its environment (phonons and electron reservoir in leads) in practice. $H_{p}$ and $H_{e p}$ stand for the phonon reservoir and its coupling to charge qubit respectively. They can be written as follows:

$$
\begin{aligned}
H_{p} & =\sum_{\mathbf{q}, \lambda} \omega_{\mathbf{q}, \lambda} b_{\mathbf{q}, \lambda}^{\dagger} b_{\mathbf{q}, \lambda} \\
H_{e p} & =\frac{1}{2} \sigma_{z} \sum_{\mathbf{q}, \lambda}\left(M_{\mathbf{q}, \lambda} b_{\mathbf{q}, \lambda}^{\dagger}+M_{\mathbf{q}, \lambda}^{*} b_{\mathbf{q}, \lambda}\right),
\end{aligned}
$$

where $b_{\mathbf{q}, \lambda}^{\dagger}\left(b_{\mathbf{q}, \lambda}\right)$ and $\omega_{\mathbf{q}, \lambda}$ are the creation (annihilation) operators and energy of the phonons with the wave vector $\mathbf{q}$ and polarization $\lambda, M_{\mathbf{q}, \lambda}$ is the electron-phonon coupling constant. The effects of the phonon bath are fully described by a spectral density

$$
J(\omega)=\sum_{\mathbf{q}, \lambda}\left|M_{\mathbf{q}, \lambda}\right|^{2} \delta\left(\omega-\omega_{\mathbf{q}, \lambda}\right) .
$$

$H_{r}$ and $H_{e r}$ in the Hamiltonian $H$ stand for the electron reservoir in leads and its coupling to charge qubit respectively.

In experiment, a pulse technique is used to switch the the $V_{s d}$ from large bias in the initialization process (an excess electron localizes in the left dot) to the zero bias in the manipulation process (the double dot is isolated from leads, and the excess electron tunnels resonantly (i.e., $\varepsilon=0$ ) back and forth between two dots) ${ }^{13}$ Restoring a large bias voltage $V_{s d}$ after the pulse time $t$ gives the measurement of dynamical elastic tunneling current which stands for the probability $n(t)$ of the excess electron in the right dot at that exact time.

Neglecting the higher order tunneling (cotunneling) between leads and the dots, the effective Hamiltonian in the manipulation process reads:

$$
H_{e f f}=T_{c} \sigma_{x}+\sum_{\mathbf{q}} \omega_{\mathbf{q}} b_{\mathbf{q}}^{\dagger} b_{\mathbf{q}}+\frac{1}{2} \sigma_{z} \sum_{\mathbf{q}}\left(M_{\mathbf{q}} b_{\mathbf{q}}^{\dagger}+M_{\mathbf{q}}^{*} b_{\mathbf{q}}\right) \cdot(6)
$$

Here, for the sake of simplicity, we omit the polarization, since it makes no difference in the theory below. When it makes difference (in Sec. IV), it will be included again and noted out explicitly. This effective Hamiltonian is the starting point for our theory.

\section{B. Theory}

The effective Hamiltonian $H_{\text {eff }}$ is equivalent to the spin-boson Hamiltonian in zero bias case. Though it seems rather simple, it cannot be solved exactly. Various analytical or numerical approaches have been proposed to obtain an approximate solution to it. ${ }^{23,24}$
Here, we apply a canonical transformation: $H^{\prime}=$ $\exp (S) H_{\text {eff }} \exp (-S)$, with the generator ${ }^{25.26 .27}$

$$
S=\sum_{\mathbf{q}} \frac{\xi_{\mathbf{q}}}{2 \omega_{\mathbf{q}}}\left(M_{\mathbf{q}} b_{\mathbf{q}}^{\dagger}-M_{\mathbf{q}}^{*} b_{\mathbf{q}}\right) \sigma_{z}
$$

Thus we get the Hamiltonian $H^{\prime}$, and decompose it into $H^{\prime}=H_{0}^{\prime}+H_{1}^{\prime}+H_{2}^{\prime}$, where

$$
\begin{aligned}
& H_{0}^{\prime}=\eta T_{c} \sigma_{x}+\sum_{\mathbf{q}} \omega_{\mathbf{q}} b_{\mathbf{q}}^{\dagger} b_{\mathbf{q}}-\sum_{\mathbf{q}} \frac{\left|M_{\mathbf{q}}\right|^{2}}{4 \omega_{\mathbf{q}}} \xi_{\mathbf{q}}\left(2-\xi_{\mathbf{q}}\right),(8) \\
& H_{1}^{\prime}=\frac{1}{2} \sigma_{z} \sum_{\mathbf{q}}\left(1-\xi_{\mathbf{q}}\right)\left(M_{\mathbf{q}} b_{\mathbf{q}}^{\dagger}+M_{\mathbf{q}}^{*} b_{\mathbf{q}}\right)+\eta T_{c} i \sigma_{y} A,(9) \\
& H_{2}^{\prime}=T_{c} \sigma_{x}(\cosh A-\eta)+T_{c} i \sigma_{y}(\sinh A-\eta A),
\end{aligned}
$$

where

$$
A=\sum_{\mathbf{q}} \frac{\xi_{\mathbf{q}}}{\omega_{\mathbf{q}}}\left(M_{\mathbf{q}} b_{\mathbf{q}}^{\dagger}-M_{\mathbf{q}}^{*} b_{\mathbf{q}}\right)
$$

and $\eta$ is a parameter which will be adjusted to minimize perturbation terms $\left(H_{1}^{\prime}\right.$ and $\left.H_{2}^{\prime}\right)$. Obviously, $H_{0}^{\prime}$ can be solved exactly. We denote the ground state of $H_{0}^{\prime}$ as

$$
|g\rangle=\left|s_{2}\right\rangle\left|\left\{0_{\mathbf{q}}\right\}\right\rangle,
$$

and the lowest excited states as

$$
\begin{aligned}
& \left|e_{s}\right\rangle=\left|s_{1}\right\rangle\left|\left\{0_{\mathbf{q}}\right\}\right\rangle, \\
& \left|e_{\mathbf{q}}\right\rangle=\left|s_{2}\right\rangle\left|1_{\mathbf{q}}\right\rangle,
\end{aligned}
$$

where $\left|s_{1}\right\rangle$ and $\left|s_{2}\right\rangle$ are eigenstates of $\sigma_{x}\left(\sigma_{x}\left|s_{1}\right\rangle=\left|s_{1}\right\rangle\right.$, $\left.\sigma_{x}\left|s_{2}\right\rangle=-\left|s_{2}\right\rangle\right),\left|\left\{0_{\mathbf{q}}\right\}\right\rangle$ stands for the vacuum state for phonon, and $\left|1_{\mathbf{q}}\right\rangle$ means that there is only 1 phonon for mode $\mathbf{q}$ and no phonon for other modes. Let $H_{1}^{\prime}|g\rangle=0$ and $\left\langle g\left|H_{2}^{\prime}\right| g\right\rangle=0$, we will get $\xi_{\mathbf{q}}$ and $\eta$ respectively as follows:

$$
\begin{aligned}
& \xi_{\mathbf{q}}=\frac{\omega_{\mathbf{q}}}{\omega_{\mathbf{q}}+2 \eta T_{c}} \\
& \eta=\exp \left[-\sum_{\mathbf{q}} \frac{\left|M_{\mathbf{q}}\right|^{2}}{2 \omega_{\mathbf{q}}^{2}} \xi_{\mathbf{q}}^{2}\right] .
\end{aligned}
$$

Now we can easily check that $\left\langle e_{s}\left|H_{1}^{\prime}\right| e_{s}\right\rangle=0$, $\left\langle e_{\mathbf{q}}\left|H_{1}^{\prime}\right| e_{\mathbf{q}}\right\rangle=0,\left\langle e_{s}\left|H_{2}^{\prime}\right| g\right\rangle=0,\left\langle e_{\mathbf{q}}\left|H_{2}^{\prime}\right| g\right\rangle=0$, and $\left\langle e_{\mathbf{q}}\left|H_{1}^{\prime}\right| e_{s}\right\rangle=V_{\mathbf{q}}$, where $V_{\mathbf{q}}=2 \eta T_{c} M_{\mathbf{q}} \xi_{\mathbf{q}} / \omega_{\mathbf{q}}$. With these relations above, we can now diagonalize the lowest excited states of $H^{\prime}$ as

$$
\begin{aligned}
H^{\prime}= & -\eta T_{c}|g\rangle\left\langle g\left|+\sum_{E} E\right| E\right\rangle\langle E| \\
& + \text { terms with high excited states. }
\end{aligned}
$$

The experiment in Ref. [13] is performed at lattice temperature below $20 \mathrm{mK}{ }^{13,14}$ At such a low temperature, 
the multiphonon process is weak enough to be negligible. So we can get the transformation as $\frac{26.27 .29}{}$

$$
\begin{aligned}
& \left|e_{s}\right\rangle=\sum_{E} x(E)|E\rangle, \\
& \left|e_{\mathbf{q}}\right\rangle=\sum_{E} y_{\mathbf{q}}(E)|E\rangle, \\
& |E\rangle=x(E)\left|e_{s}\right\rangle+\sum_{\mathbf{q}} y_{\mathbf{q}}(E)\left|e_{\mathbf{q}}\right\rangle,
\end{aligned}
$$

where

$$
\begin{aligned}
& |x(E)|^{2}=\left[1+\sum_{\mathbf{q}} \frac{\left|V_{\mathbf{q}}\right|^{2}}{\left(E+\eta T_{c}-\omega_{\mathbf{q}}\right)^{2}}\right]^{-1}, \\
& \left|y_{\mathbf{q}}(E)\right|^{2}=\frac{\left|V_{\mathbf{q}}\right|^{2}}{\left(E+\eta T_{c}-\omega_{\mathbf{q}}\right)^{2}}|x(E)|^{2},
\end{aligned}
$$

and the E's are solutions to the equation

$$
E-\eta T_{c}-\sum_{\mathbf{q}} \frac{\left|V_{\mathbf{q}}\right|^{2}}{E+\eta T_{c}-\omega_{\mathbf{q}}}=0 .
$$

The population inversion can be defined as $P(t)=$ $\left\langle\psi(t)\left|\sigma_{z}\right| \psi(t)\right\rangle$, where $|\psi(t)\rangle$ is the total wavefunction (qubit and reservoir) in Schrödinger picture, and

$$
|\psi(t)\rangle=e^{-S} e^{-i H^{\prime} t} e^{S}|\psi(0)\rangle .
$$

Since the qubit is initialized at the state $|L\rangle$, it is reasonable to choose $|\psi(0)\rangle=e^{-S}|L\rangle\left|\left\{0_{\mathbf{q}}\right\}\right\rangle$. Then we can obtain

$$
\begin{aligned}
P(t)= & \left\langle\left\{0_{\mathbf{q}}\right\}\left|\left\langle L\left|e^{i H^{\prime} t} e^{S} \sigma_{z} e^{-S} e^{-i H^{\prime} t}\right| L\right\rangle\right|\left\{0_{\mathbf{q}}\right\}\right\rangle \\
= & -\frac{1}{2} \sum_{E}|x(E)|^{2} \exp \left[-i\left(E+\eta T_{c}\right) t\right]-\frac{1}{2} \sum_{E}|x(E)|^{2} \exp \left[i\left(E+\eta T_{c}\right) t\right] \\
= & -\frac{1}{4 \pi i} \oint_{C} d \omega e^{-i \omega t}\left(\omega-2 \eta T_{c}-\sum_{\mathbf{q}} \frac{\left|V_{\mathbf{q}}\right|^{2}}{\omega+i 0^{+}-\omega_{\mathbf{q}}}\right)^{-1} \\
& -\frac{1}{4 \pi i} \oint_{C^{\prime}} d \omega e^{i \omega t}\left(\omega-2 \eta T_{c}-\sum_{\mathbf{q}} \frac{\left|V_{\mathbf{q}}\right|^{2}}{\omega-i 0^{+}-\omega_{\mathbf{q}}}\right)^{-1},
\end{aligned}
$$

where $\omega=E+\eta T_{c}$. Denoting the real and imaginary part of $\sum_{\mathbf{q}}\left|V_{\mathbf{q}}\right|^{2} /\left(\omega \pm i 0^{+}-\omega_{\mathbf{q}}\right)$ as $R(\omega)$ and $\mp \gamma(\omega)$ respectively, we can get

$$
\begin{aligned}
R(\omega) & =\sum_{\mathbf{q}} \mathcal{P} \frac{\left|V_{\mathbf{q}}\right|^{2}}{\omega-\omega_{\mathbf{q}}} \\
& =4\left(\eta T_{c}\right)^{2} \mathcal{P} \int_{0}^{\infty} d \omega^{\prime} \frac{J\left(\omega^{\prime}\right)}{\left(\omega-\omega^{\prime}\right)\left(\omega^{\prime}+2 \eta T_{c}\right)^{2}} \\
\gamma(\omega) & =\pi \sum_{\mathbf{q}}\left|V_{\mathbf{q}}\right|^{2} \delta\left(\omega-\omega_{\mathbf{q}}\right) \\
& =4 \pi\left(\eta T_{c}\right)^{2} \frac{J(\omega)}{\left(\omega+2 \eta T_{c}\right)^{2}},
\end{aligned}
$$

where $\mathcal{P}$ stands for Cauchy principal value, and the spectral density $J(\omega)$ is defined in Eq. (5). The parameter $\eta$ determined by Eq. (15) and Eq. (16) can also be expressed as

$$
\eta=\exp \left[-\int_{0}^{\infty} d \omega \frac{J(\omega)}{2\left(\omega+2 \eta T_{c}\right)^{2}}\right] .
$$

The contour integral in Eq. (26) can proceed by calcu- lating the residue of integrand and the result is

$$
P(t)=-\cos \left(\omega_{r} t\right) \exp (-\gamma t)
$$

where we have applied the second order approximation ${ }^{26}$

$$
\gamma \simeq \gamma\left(2 \eta T_{c}\right)=\frac{1}{4} \pi J\left(2 \eta T_{c}\right),
$$

and $\omega_{r}$ is the solution to the equation

$$
\omega-2 \eta T_{c}-R(\omega)=0 .
$$

Finally, the tunneling electron population (probablity) in the right dot at time $t$ is given by

$$
n(t)=\frac{1}{2}[1+P(t)]=\frac{1}{2}\left[1-\cos \left(\omega_{r} t\right) \exp (-\gamma t)\right] .
$$

Thus a rather simple expression for the dynamical tunneling is obtained analytically. The damped oscillation indicated by this expression agrees with the experiment in Ref. [13]. 

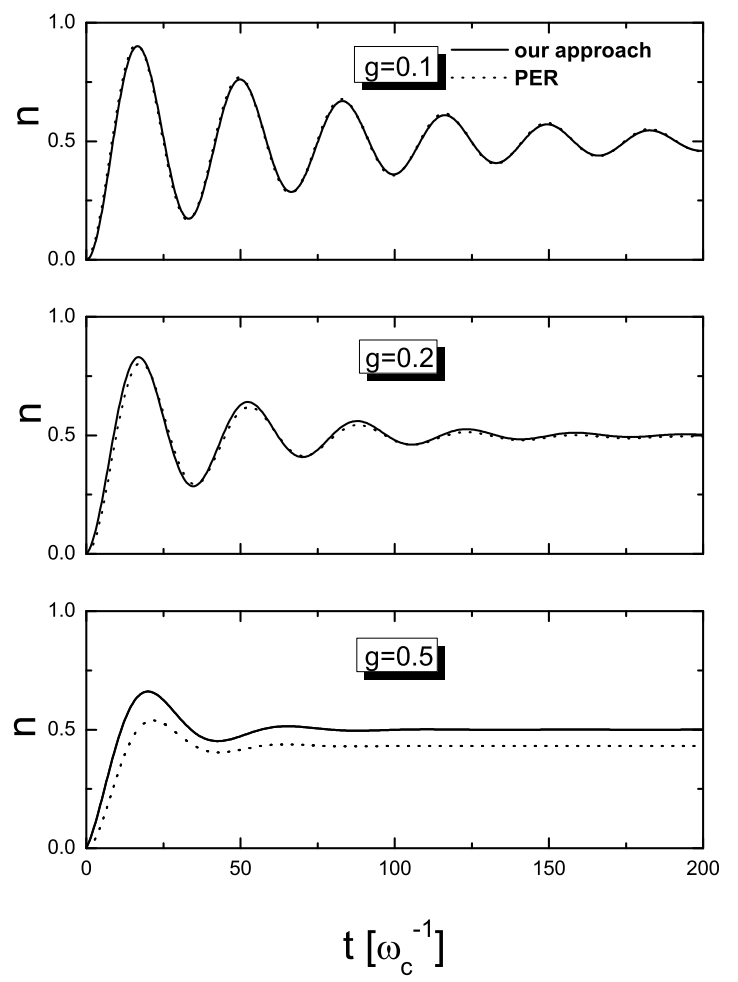

FIG. 1: The tunneling electron population in the right dot as a function of time for Ohmic dissipation at low temperature limit with the coupling constant $g=0.1,0.2$, and 0.5 . The resonant tunneling rate is fixed as $T_{c}=0.1 \omega_{c}$. The solid curve and dot curve stand for the results of our approach and PER approach respectively.

\section{COMPARISON WITH PER APPROACH}

As mentioned in the introduction above, Brandes has also derived an expression for the dynamical tunneling in the double-dot system. In his derivation the BornMarkov approximation is made and the electron-phonon coupling $H_{e p}$ is treated as a peterbation (PER) ${ }^{22}$ In this section, we give a comparison between the PER and our approach.

The spectral density $J(\omega)$ defined in Eq. (5) is the only quantity describing the interaction between the system and its environment that enters into the dynamical tunneling. To get the final result, we need the knowledge of this spectral function first. Ordinarily, it can be written in a power law form with a cutoff $\omega_{c}$,

$$
J(\omega)=g \omega^{s} e^{-\omega / \omega_{c}},
$$

where $0<s<1, s=1$, and $s>1$ corresponds to the subOhmic, Ohmic, and super-Ohmic spectral, respectively, $\mathrm{g}$ is a coupling constant. Since the result for $n(t)$ is only presented for the Ohmic case in Ref. [22], we also use the Ohmic spectral in this section for convenience. We
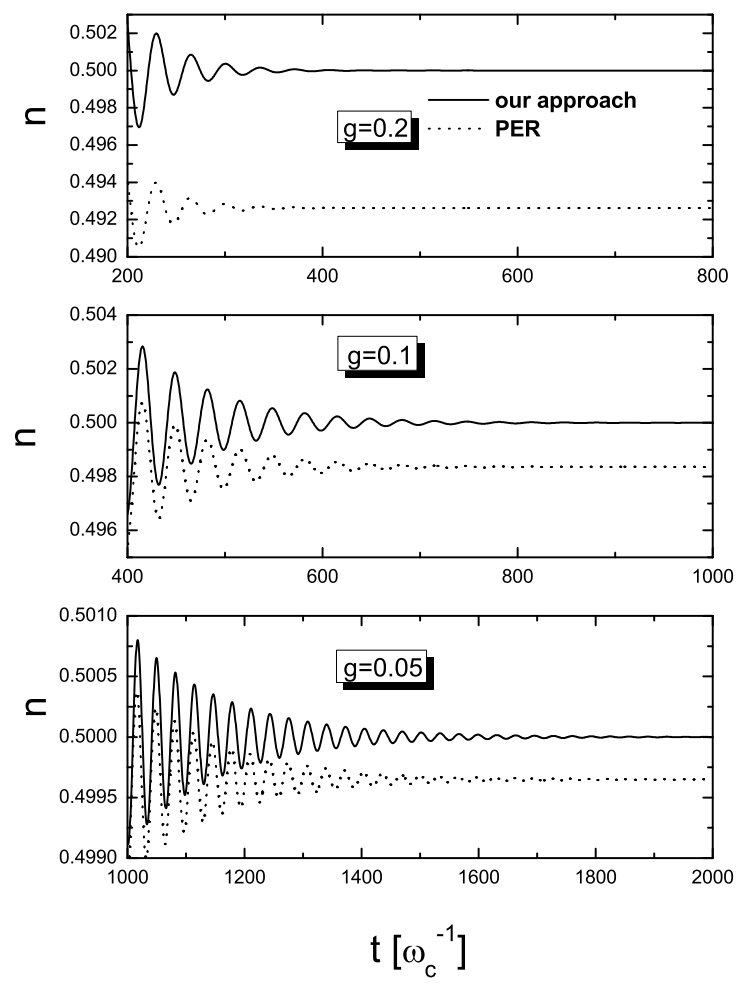

FIG. 2: Long time behavior of the tunneling electron population in the right dot as a function of time for Ohmic dissipation at low temperature limit with the coupling constant $g=0.2,0.1$, and 0.05 . Others are the same as Fig. 1 .

should point out that, our approach is not limited by the form of $J(\omega)$. However, the Born-Markov approximation made in PER approach is only meaningful and defined for $s \geq 1.22$

Fig. 1 presents the time evolution of the tunneling electron population (probability) in the right dot with three different coupling constants. The result of both our approach and PER is given at the temperature $T=20 \mathrm{mK}$, at which the experiment is performed 13 . The result at higher temperature can also be obtained by PER and we find that the difference is indistinguishable when the temperature is below $0.1 \mathrm{~K}$, which testifies the validity of the single phonon process assumption in the transformation in Eq. (18), (19), and (20). The comparison shows that, in weak coupling regime $(g \leq 0.2)$, the results of two approaches agree with each other well; while in strong coupling regime $(g \sim 0.5)$, the difference between two approaches becomes clear. Although both two approaches are based on perturbation theory, the perturbations in two approaches are different. The PER approach treats the coupling $H_{e p}$ to the phonon reservoir as a perturbation, which consequently requires the coupling constant $g$ to be restricted in the weak coupling regime. In our approach, the perturbation theory is exerted to the Hamil- 
tonian after a canonical transformation. The perturbation is taken as $H_{1}^{\prime}$ and $H_{2}^{\prime}$, which can be minimized by the variational parameter $\eta$. So our approach can be easily extended to the strong coupling regime (it works well for the whole range of $0<g<2) 26$

Then we focus on the long time behavior of the time evolution. Since the double dot is symmetric (i.e., unbiased case, $\varepsilon=0$ ), the population in two dots at long time limit should also be symmetric. In other words, the asymptotic value of $n(t)$ is expected to be 0.5 . But in the strong coupling case $(g=0.5)$ of Fig. 1 , it has shown that there is a gap between the result of PER at long time limit and that of our approach which equals 0.5. Actually, even in weak coupling regime, this kind of deviation still exists but rather small, as can be seen from Fig. 2 in an enlarged view for coupling constant $g=0.2,0.1$, and 0.05 . Since the asymptotic value from non-Markovian calculation presented by DiVincenzo and Loss also equals $0.5,28$ the deviation in PER approach must arise from Markov approximation. And it is avoided in our approach.

There are two alternative ways of perturbation theory: one is in the coupling $H_{e p}$ (PER), another is in the interdot coupling $T_{c}$ (POL) ${ }^{22}$ To get a good approximation, one must restrict the PER approach in the weak coupling regime, as analyzed above. But it does not mean there is no requirement for $T_{c}$. In Fig. 3, we present the dynamical population in the right dot $n(t)$ in weak coupling $(g=0.1)$, but with three different int-dot tunneling rates $T_{c}=0.05,0.01$, and $0.001 \omega_{c}$. We find that, in strong inter-dot tunneling case $\left(T_{c}>0.05 \omega_{c}\right)$, two approaches give consistent results; while in weak tunneling case $\left(T_{c}<0.01 \omega_{c}\right)$, the results deviate from each other. It is well known that the energy difference for the two eigenstates (i.e., the bonding and anti-bonding states) of the double dot system is the Rabi splitting $\Delta=\sqrt{\varepsilon^{2}+4 T_{c}^{2}}$. Here in the unbiased case $(\varepsilon=0)$, the energy difference $\Delta$ is determined only by the inter-dot tunneling $T_{c}$. When $T_{c}$ is too small, the energy difference $\Delta$ between two states may be smaller than the perturbation $H_{e p}$ of the PER approach. In such situation, the energy states are nearly-degenerate and the perturbation theory for a nondegenerate state of the PER approach is not suitable. So the PER breaks down in weak inter-dot tunneling regime. In our approach, however, the perturbation terms $\left(H_{1}^{\prime}\right.$ and $\left.H_{2}^{\prime}\right)$ decrease with $T_{c}$, thus the perturbation theory always works well for the whole range of $T_{c}$.

\section{PHONON INDUCED DECOHERENCE}

One of the central points in quantum physics is the loss of coherence of the quantum system. In this section, the spectral density for the double-dot system coupling to bulk phonons is derived from the microscopic view. Then the phonon induced decoherence is analyzed at length. And the suppression of decoherence is discussed.
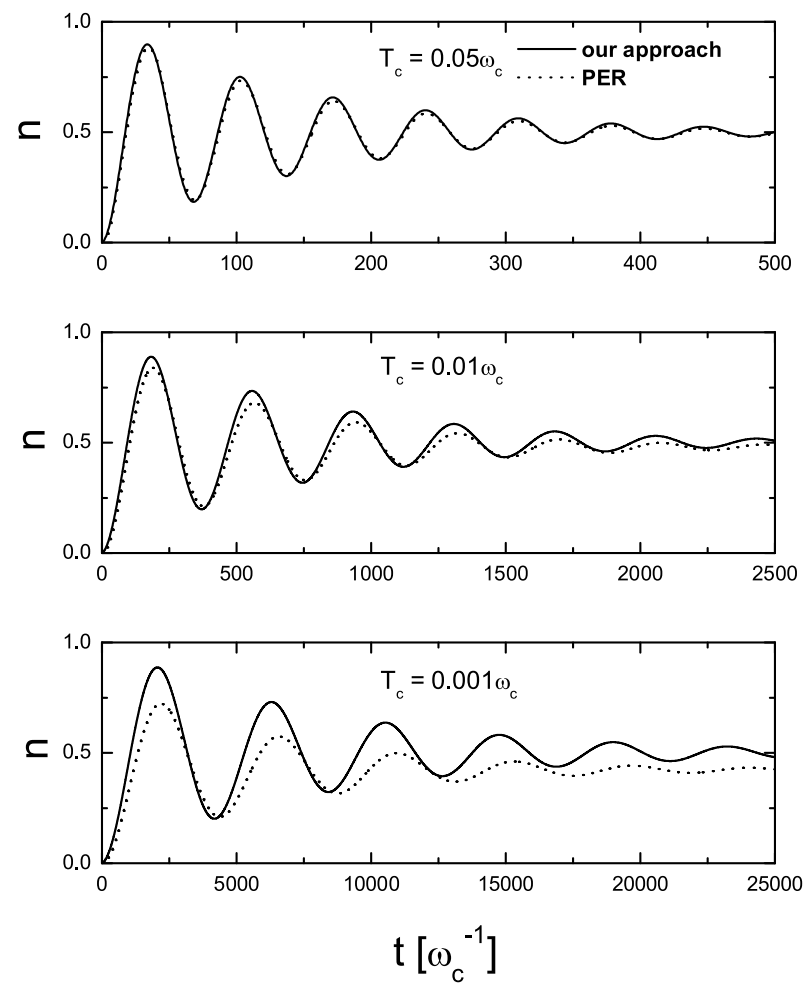

FIG. 3: The tunneling electron population in the right dot as a function of time for Ohmic dissipation at low temperature limit with the tunneling rate $T_{c}=0.05,0.01$, and $0.001 \omega_{c}$. The coupling constant is fixed as $g=0.1$.

\section{A. Spectral density}

In last section, we use the Ohmic spectral to calculate the dynamical tunneling current. Obviously, it is not appropriate to describe the properties of the double-dot system. Since all relevant information of the environment and its coupling to the qubit system is contained in the spectral function, it is necessary and important to obtain a suitable spectral function to describe a double quantum dot. Here, we only consider the coupling to the bulk acoustic phonons, because we are interested in the low temperature limit case. The only two types of interaction between electrons and acoustic phonons in semiconductors are piezoelectric coupling and deformation potential coupling.

We assume the electron wave functions $|L\rangle$ and $|R\rangle$ are sharply around the center of dot with Gaussian shape $\sim$ $\exp \left[-r^{2} /\left(2 l^{2}\right)\right]$, where $l$ is the the dot size. One can show that the piezoelectric coupling constant for GaAs (zincblende structure) is 20

$$
\begin{aligned}
M_{\mathbf{q}, \lambda}^{\mathrm{pz}}= & -\left(\frac{1}{2 \rho q s_{\lambda} V}\right)^{1 / 2} M e^{-a^{2} q^{2} / 4} \\
& \times\left(\xi_{1}^{\lambda} e_{2} e_{3}+\xi_{2}^{\lambda} e_{1} e_{3}+\xi_{3}^{\lambda} e_{1} e_{2}\right) \sin \left(\frac{\mathbf{q} \cdot \mathbf{d}}{2}\right),
\end{aligned}
$$

where $\rho$ is the density of the crystal, $V$ is normalizing vol- 
ume, $s$ is the sound velocity in crystal (longitudinal sound and transverse sound have different velocities), $e_{i}=q_{i} / q$, $\xi$ is the polarization vector whose components depend on the polarization mode $\lambda, M$ is the piezoconstant, and $d$ is the center-to-center distance between two dots. Here, we include the polarization again, since different polarization modes give different coupling constants. With the simple dispersion relation $\omega_{\mathbf{q}, \lambda}=s_{\lambda} q$, one can now calculate, according to the definition in Eq. (5), the spectral function $J^{\mathrm{pz}}(\omega)$ due to piezoelectric coupling. But the expression is rather complicated. To obtain a tractable form of the piezoelectric coupling we use the angular average following Bruus et al ${ }^{30}$ and Brandes et al ${ }^{15}$. Then we get

$$
J^{\mathrm{pz}}(\omega)=g_{\mathrm{pz}} \omega\left(1-\frac{\omega_{d}}{\omega} \sin \frac{\omega}{\omega_{d}}\right) e^{-\omega^{2} / 2 \omega_{l}^{2}},
$$

where $\omega_{d}=s / d, \omega_{l}=s / l$, and $g_{\mathrm{pz}}=P / 2 \pi^{2} \rho s^{3}$ with

$$
P=M^{2}\left(\frac{12}{35}+\frac{1}{x} \frac{16}{35}\right)
$$

where the transverse sound velocity is expressed as $x$ times the longitudinal sound velocity $s$. For the deformation potential coupling, the contribution from TAacoustic phonons is small enough to be neglected as compared with that from LA-acoustic phonons. So the coupling constant can be written $\operatorname{as}^{20}$

$$
M_{\mathbf{q}}^{\mathrm{df}}=i q \Xi\left(\frac{1}{2 \rho q s V}\right)^{1 / 2} e^{-a^{2} q^{2} / 4} \sin \left(\frac{\mathbf{q} \cdot \mathbf{d}}{2}\right),
$$

where $\Xi$ is the deformation potential. Then we can easily get the spectral function due to deformation coupling

$$
J^{\mathrm{df}}(\omega)=g_{\mathrm{df}} \omega^{3}\left(1-\frac{\omega_{d}}{\omega} \sin \frac{\omega}{\omega_{d}}\right) e^{-\omega^{2} / 2 \omega_{l}^{2}},
$$

where $g_{\mathrm{df}}=\Xi^{2} / 8 \pi^{2} \rho s^{5}$.

With the parameters of GaAs in Ref. [30], we can estimate that $g_{\mathrm{pz}} \approx 0.035$ and $g_{\mathrm{df}} \approx 0.029(p s)^{-2}$. Previous work states that the contribution from deformation potential phonons is small as compared with piezoelectric phonons in double-dot system of GaAs material. ${ }^{22}$ Our result also proves it to be true in the weak confinement regime (large dot size). But it is not valid when the dot size is decreased to the strong confinement regime. Fig. 4 shows the spectral functions $J^{\mathrm{pz}}(\omega)$ and $J^{\mathrm{df}}(\omega)$ in strong confinement regime, with $\omega_{l}=1(\mathrm{ps})^{-1}$ (i.e., dot size $l=5 \mathrm{~nm}$ ) and $\omega_{l}=0.5(\mathrm{ps})^{-1}$ (i.e., dot size $l=10 \mathrm{~nm}$ ). As we can see, $J^{\mathrm{df}}(\omega)$ is comparable to $J^{\mathrm{pz}}(\omega)$ in that regime. But it shrinks much faster than $J^{\mathrm{pz}}(\omega)$ as the dot size is increased and is negligible when the dot size $l>50 \mathrm{~nm}$. Fig. 4 also shows the spectral functions $J^{\mathrm{pz}}(\omega)$ and $J^{\mathrm{df}}(\omega)$ at two different center-to-center distances, with $\omega_{d}=0.05(\mathrm{ps})^{-1}$ (i.e., $d=100 \mathrm{~nm}$ ) and $\omega_{d}=0.02(\mathrm{ps})^{-1}$ (i.e., $\left.d=250 \mathrm{~nm}\right)$. The influence from the parameter $d$ to both $J^{\mathrm{pz}}(\omega)$ and $J^{\mathrm{df}}(\omega)$ is small compared with that from the parameter $l$. It adds the spectral function an oscillation term, and the oscillation frequency (determined by $\omega_{d}$ ) is increased with $d$. All these
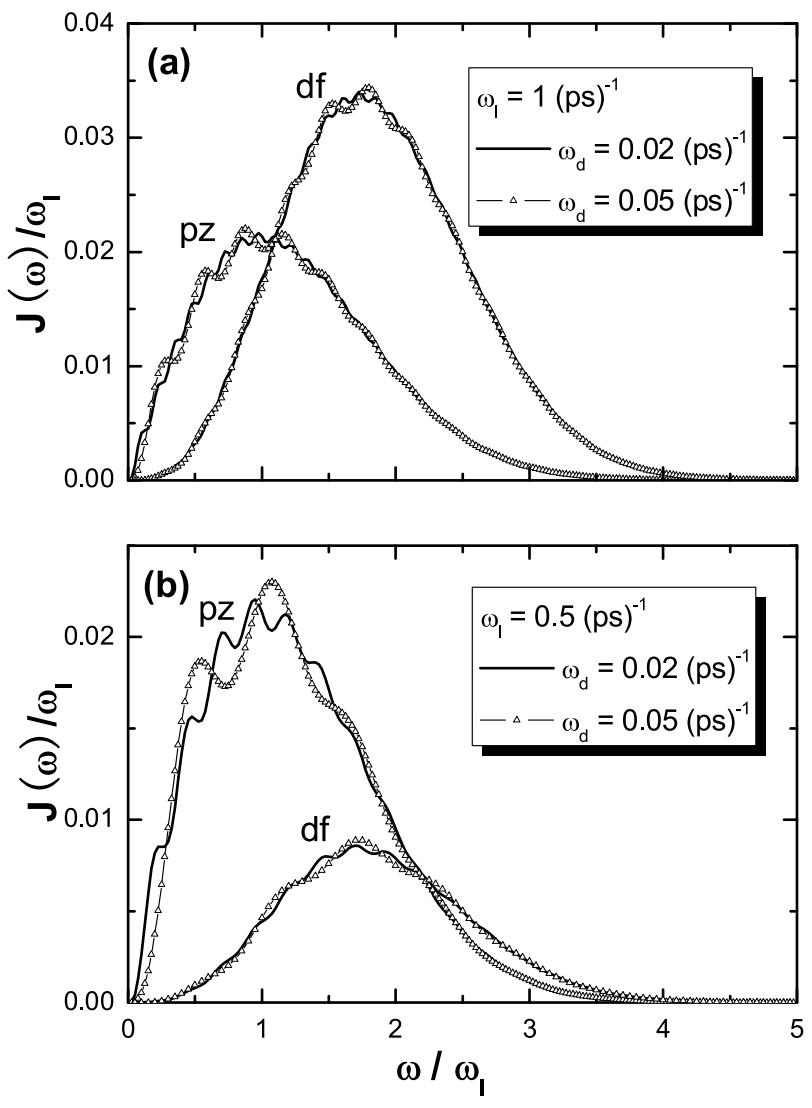

FIG. 4: Spectral functions of double quantum dot due to piezoelectric coupling (labelled by pz) and deformation potential coupling (labelled by df) with $\omega_{d}=0.02$ and $\omega_{d}=$ $0.05(\mathrm{ps})^{-1}$. (a) $\omega_{l}=1(\mathrm{ps})^{-1}$. (b) $\omega_{l}=0.5(\mathrm{ps})^{-1}$.

properties of spectral functions $J^{\mathrm{pz}}(\omega)$ and $J^{\mathrm{df}}(\omega)$ determine the decoherence induced by piezoelectric coupling and deformation potential coupling to phonons, respectively.

As one can see in Eq. (35) and Eq. (38), the deformation potential coupling constant is real, while the piezoelectric coupling constant is imaginary, which means they do not interfere ${ }^{30.31}$ Thus the total spectral density is

$$
J(\omega)=J^{\mathrm{pz}}(\omega)+J^{\mathrm{df}}(\omega) .
$$

\section{B. Decoherence induced by acoustic phonons}

The decoherence of quantum system due to interacting with environment is a crucial point in quantum information. In a double quantum dot, scattering by phonons can cause considerable loss of coherence accompanied by dissipation when the tunneling electron flips back and forth between two dots. One of the advantages of our approach is that the decoherence rate in the this process is obtained explicitly. Thus one can analyze it clearly.

Using the expressions of spectral density $J^{\mathrm{pz}}(\omega)$ (Eq. 

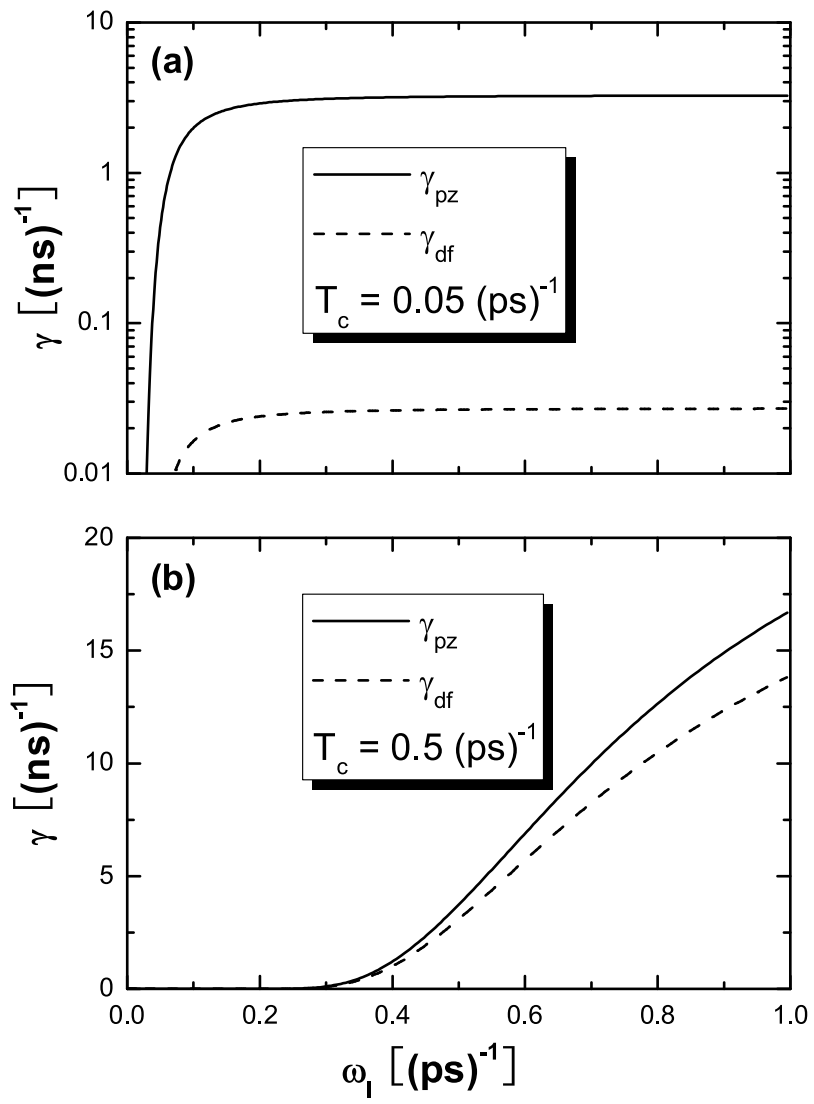

FIG. 5: Decoherence rates $\gamma_{\mathrm{pz}}$ (solid line) and $\gamma_{\mathrm{df}}$ (dash line) as functions of cutoff frequency $\omega_{l}$ when $\omega_{d}=0.02(\mathrm{ps})^{-1}$. The tunneling rates in Fig.5 (a) and Fig.5 (b) are 0.05 (ps) ${ }^{-1}$ and $0.5(\mathrm{ps})^{-1}$, respectively.

(36)) and $J^{\mathrm{df}}(\omega)($ Eq. (39)) above, the decoherence rates induced by piezoelectric and deformation potential coupling are written as

$$
\gamma_{\mathrm{pz}}=\frac{1}{2} \pi g_{\mathrm{pz}} \eta T_{c}\left(1-\frac{\omega_{d}}{2 \eta T_{c}} \sin \frac{2 \eta T_{c}}{\omega_{d}}\right) e^{-2 \eta^{2} T_{c}{ }^{2} / \omega_{l}{ }^{2}},
$$

and

$$
\gamma_{\mathrm{df}}=2 \pi g_{\mathrm{df}} \eta^{3} T_{c}^{3}\left(1-\frac{\omega_{d}}{2 \eta T_{c}} \sin \frac{2 \eta T_{c}}{\omega_{d}}\right) e^{-2 \eta^{2} T_{c}{ }^{2} / \omega_{l}{ }^{2}},
$$

respectively. Here, one should note the parameter $\eta$ in Eq. (41) and that of the Eq. (42) are not the same, because they are calculated from Eq. (29) with different spectral functions $\left(J^{\mathrm{pz}}(\omega)\right.$ and $J^{\mathrm{df}}(\omega)$, respectively). According to Eq. (40), the total decoherence rate induced by acoustic phonons is $\gamma=\gamma_{\mathrm{pz}}+\gamma_{\mathrm{df}}$.

Fig. 5 presents the decoherence rates $\gamma_{\mathrm{pz}}$ and $\gamma_{\mathrm{df}}$ as functions of $\omega_{l}$ at two different tunneling rates $T_{c}=$ $0.05(\mathrm{ps})^{-1}$ and $T_{c}=0.5(\mathrm{ps})^{-1}$. Another parameter $\omega_{d}$ is fixed as $0.02(\mathrm{ps})^{-1}$, which means the center-to-center distance between two dots is about $250 \mathrm{~nm}$. As showed by Fig. 5(a), at small tunneling rate, the contribution to decoherence rate arose from deformation potential coupling

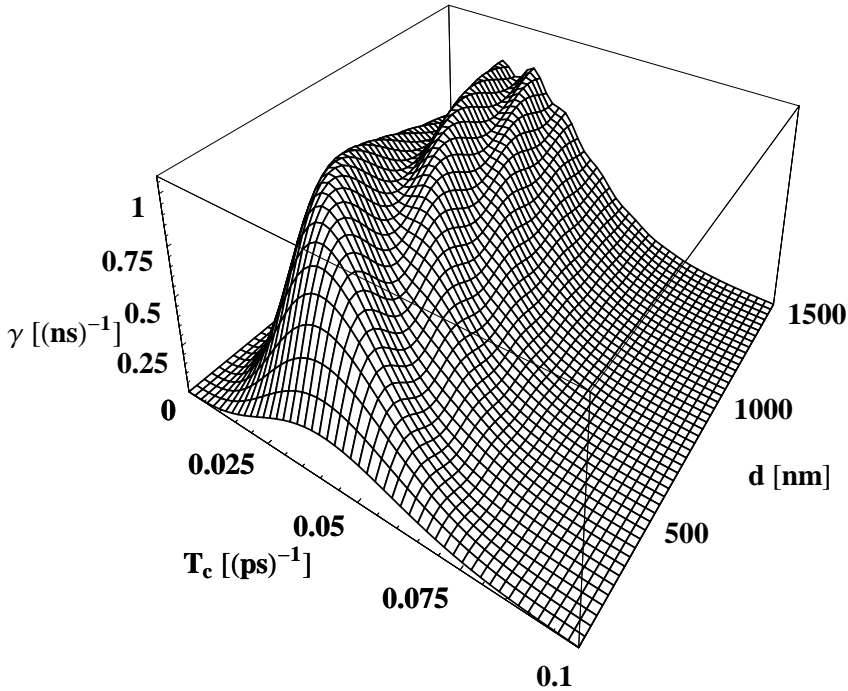

FIG. 6: Decoherence rate $\gamma$ as a function of tunneling rate $T_{c}$ and distance between two dots $d$. The dot size is chosen to be $100 \mathrm{~nm}$.

is small compared with that from piezoelectric coupling, even in strong confinement regime (i.e., $\omega_{l} \sim 1(\mathrm{ps})^{-1}$ ). But when the tunneling rate is large (Fig. 5(b)), the decoherence rates arising from these two mechanisms are comparable, thus the contribution from the deformation potential coupling can not be neglected. Fig. 5(a) and (b) also show that the decoherence rates (both $\gamma_{\mathrm{pz}}$ and $\left.\gamma_{\mathrm{df}}\right)$ are suppressed when $\omega_{c}$ is decreased, which indicates that one should use large dot size to get little decoherence. However, large dot size means small characteristic energy spacing (on-site charging energy) $\omega_{01}$ of single quantum dot. It is well known that, our two level Hamitonian is valid to describe the double-dot system only if $T_{c}, k_{B} T \ll \omega_{01}$, where $k_{B}$ is Boltzmann constant ${ }^{32}$ So, low temperature technique is needed to maintain good quantum properties of dots when the dot size is large, just as the experiment is performed 13

In what follows, we choose a large dot size of $100 \mathrm{~nm}$ (approximate size for the dot in Ref. [13]), i.e., $\omega_{l}=$ $0.05(\mathrm{ps})^{-1}$. Since the tunneling barriers in experiment of Ref. [13] are made by depleting electrons with negative gate voltage, their tunneling rates are flexible ${ }^{33}$ In Fig. 6 , we present the decoherence rate $\gamma\left(\approx \gamma_{\mathrm{pz}}\right.$ at that dot size) as a function of tunneling rate and distance between two dots (from 100 to $1500 \mathrm{~nm}$ ). Some oscillations, coming from the sine term in the spectral density, are discerned from this 3-dimensional figure. We find the characteristic decoherence time $T_{2}=1 / \gamma$ speculated from the figure is about $1 \mathrm{~ns}$, which corresponds well with the value fitted from the experimental curve 13 So the coupling to phonons is one of the main decoher- 
ence mechanisms in such a double-dot system. It also shows that the decoherence rate increases with tunneling rate $T_{c}$ when $T_{c}<\omega_{l}$. But larger tunneling rate will suppress the decoherence drastically, even to zero when $T_{c} \approx 0.1$ (ps) $)^{-1}$ (i.e., $\left.2 \omega_{l}\right)$. This value of $T_{c}$ is still in the range of $\ll \omega_{01}=1.3 \mathrm{meV} \sim 2(\mathrm{ps})^{-1},{ }^{13}$ in which our two level model holds. Thus, such kind of decoupling mechanism can be probably realized.

\section{CONCLUSION}

In conclusion, we have investigated the charge qubit dynamics in a semiconductor double quantum dot coupled to phonons at low temperature limit. Our approach is a perturbation theory after a unitary transformation. The dynamical tunneling current is obtained explicitly as a simple damped Rabi oscillation. The comparison with PER approach shows the advantages of our approach is that: it is not restricted by the form of spectral density; it can be extended to strong coupling regime and works well for the whole range of tunneling rate $T_{c}$; the long time behavior is also consistent with the symmetric double-dot system. Additionally, the simple decoherence rate expression allows us to analyze the phonon induced decoherence clearly. We find that, in strong confinement regime of dot and large tunneling rate $T_{c}$ $\left(>0.1(\mathrm{ps})^{-1}\right)$, the contribution to decoherence from deformation potential coupling can not be neglected compared to that from piezoelectric coupling in GaAs material. The decoherence arose from both these two mechanism will be suppressed when the dot size is increased. The decoupling with phonons will happen when the condition $2 \omega_{l}<T_{c} \ll \omega_{01}$ is met.

Finally, we hope our predictions can be testified by experiment in the near future.

We thank T.Fujisawa for helpful discussions and careful reading of the manuscript. This work was partly supported by National Natural Science Foundation of China (No.10274051) and Shanghai Natural Science Foundation (No.03ZR14060).
* Electronic address: wuzj@sjtu.edu.cn

1 D. Bouwmeester, A. K. Ekert, and Zeilinger, The Physics of Quantum Information (Springer-Verlag, Berlin, 2000).

2 M. A. Nielsen and I. L. Chuang, Quantum Computation and Quantum Information (Cambridge University Press, UK, 2000).

3 C. Monroe, Nature (London) 416, 238(2002).

4 D. Loss and D. P. DiVincenzo, Phys. Rev. A 57, 120 (1998).

5 B. E. Kane, Nature (London) 393, 133 (1998).

6 Y. Nakamura, Yu. A. Pashkin, and J. S. Tsai, Nature (London) 398, 786 (1999).

7 E. Bibow, P. Lafarge, and L. P. Lvy, Phys. Rev. Lett. 88, 017003 (2002)

8 A. Zrenner, E. Beham, S. Stufler, F. Findeis, M. Bichler, G. Abstreiter, Nature (London) 418, 612 (2002).

9 T. Fujisawa, T. H. Oosterkamp, W. G. van der Wiel, B. W. Broer, R. Aguado, S. Tarccha, L. P. Kouwenhoven, Science 282, 932 (1998).

10 T. H. Oosterkamp, T. Fujisawa, W. G. van der Wiel, K. Ishibashi, R. V. Hijman, S. Tarucha, L. P. Kouwenhoven, Nature 395, 873 (1998).

11 S. Gardelis, C. G. Smith, J. Cooper, D. A. Ritchie, E. H. Linfield, Y. Jin, and M. Pepper, Phys. Rev. B 67, 073302 (2003).

12 L. C. L. Hollenberg, A. S. Dzurak, C. Wellard, A. R. Hamilton, D. J. Reilly, G. J. Milburn, and R. G Clark, Phys. Rev. B 69, 113301, (2004).

13 T. Hayashi, T. Fujisawa, H. D. Cheong, Y. H. Jeong, and Y. Hirayama, Phys. Rev. Lett. 91, 226804 (2003).

14 T. Fujisawa, T. Hayashi, and Y. Hirayama, J. Vac. Sci. Technol. B 22, 4 (2004).

15 T. Brandes and B. Kramer, Phys. Rev. Lett. 83, 3021 (1999).
16 T. Brandes and T. Vorrath, Phys. Rev. B 66, 075341 (2002).

17 R. Aguado and T. Brandes, Eur. Phys. J. B. 40, 357 (2004).

18 R. Aguado and T. Brandes, Phys. Rev. Lett. 92, 206601 (2004).

19 T. Brandes, R. Aguado, and G. Platero Phys. Rev. B 69, 205326 (2004).

${ }^{20}$ L. Fedichkin and A. Fedorov, Phys. Rev. A 69, 032311 (2004).

21 L. Fedichkin, A. Fedorov, IEEE Trans. Nanotechn., in press.

22 T. Brandes, arXiv:cond-mat/0409771

23 A. J. Leggett, S. Chakravarty, A. T. Dorsey, M. P. A. Fisher, A. Garg, and W. Zwerger, Rev. Mod. Phys. 59, 1 (1987).

24 U. Weiss, Quantum Dissipative System (World scientific, Singapore, 1993).

25 R. Silbey and R. A. schoeller, J. Chem. Phys. 80, 2615 (1984).

26 H. Zheng, Eur. Phys. J. B 38, 559 (2004).

27 Z. J. Wu, K. D. Zhu, and Hang Zheng, Phys. Lett. A 333, 310 (2004).

28 D. P. DiVincenzo and D. Loss, arXiv:cond-mat/0405525

29 F. Guinea, V. Hakim, and A. Muramatsu, Phys. Rev. B 32, 4410 (1985).

${ }^{30}$ H. Bruus, K. Flensberg, and H. Smith, Phys. Rev. B 48, 11144 (1993).

31 G. D. Mahan, Many-Particle Physics (Plenum Press, New York, 1990).

32 S. D. Barrett and G. J. Milburn, Phys. Rev. B 68, 155307 (2003).

33 T. Fujisawa (private communication). 